\title{
Lack of nano size effect on electrochemistry of dopamine at a gold nanoparticle modified indium tin oxide electrode
}

\author{
HUANG RongFu \& GUO Liang-Hong * \\ State Key Laboratory of Environmental Chemistry and Ecotoxicology; Research Center for Eco-environmental Sciences, \\ Chinese Academy of Sciences, Beijing 100085, China
}

\begin{abstract}
Nanometer sized materials have been shown to possess excellent chemical and electrochemical catalytic properties. In this work, a gold nanoparticle (AuNP) modified indium tin oxide (ITO) electrode was employed for investigating its electro-catalytic property. AuNP was deposited on the 3-aminopropyltriethoxysilane (APTES) modified ITO electrode by self-assembly, and was characterized by scanning electron microscopy and cyclic voltammetry. Although the electrochemical reaction of dopamine was very sluggish on the ITO/APTES electrode, it was significantly enhanced after AuNP deposition. The cyclic voltammogram exhibited apparent dependence on the surface coverage of $11 \mathrm{~nm}$ AuNPs, which could be rationalized by different modes of mass diffusion. Among the different sizes of AuNP investigated, the lowest anodic peak potential was observed on $11 \mathrm{~nm}$ AuNP. However, the potential was still about $50 \mathrm{mV}$ more positive than that obtained on a bulk gold electrode of similar geometry. It is therefore concluded that there is no nanometer size effect of AuNP modified ITO on the electrochemistry of dopamine.
\end{abstract}

gold nanoparticle, dopamine, electrocatalysis, indium tin oxide

\section{Introduction}

Nanomaterials possess extraordinary chemical, electronic and optical properties which are unique to their nanometer size. Among them, metal nanoparticles have shown excellent catalytic properties towards the redox reaction of significant chemical and biochemical molecules such as dopamine, ascorbic acid, $\mathrm{NADH}$, glucose, $\mathrm{H}_{2} \mathrm{O}_{2}$, oxygen and $\mathrm{NO}$ [1-3]. On metal nanoparticle based electrodes, the redox reaction is significantly facilitated, exhibiting reduced overpotential and improved reversibility $[4,5]$.

In the field of electroanalysis with metal nanoparticles, gold nanoparticles (AuNPs) are widely used due to their excellent electrical conductivity, ease of synthesis, and established surface chemistry. Although gold is relatively in-

*Corresponding author (email: lhguo@ rcees.ac.cn) ert in the bulk form, gold nanoparticles exhibit high electrochemical reactivity. Gold nanoparticles based electrodes are mostly fabricated on conventional conductive substrates such as gold, carbon and indium tin oxide (ITO). The nanoparticles could be produced on the substrate surface by in situ chemical or electrochemical synthesis. This approach ensures intimate contact between the nanoparticle and base electrode, but may suffer from weak adhesion. Alternatively, the nanoparticles could be synthesized ex situ, and then attached to a base electrode by covalent or physical assembly. For instance, AuNPs were deposited in situ on a gold wire electrode by electrochemical reduction. Enhanced cathodic reaction of oxygen was observed on the nanoparticle-modified electrode over the bulk gold [6]. Pre-synthesized AuNPs were attached to gold disc electrodes via thiol linkers and utilized in the detection of dopamine [7], epinephrine [8], catechol [9], as well as in the electrochemical reduction of oxygen [10]. Gold nanoparticles were also loaded into a sol- 
gel film on a gold substrate to form a 3D nanoparticle assembly, and employed in the continuous monitoring of hydrazine [11]. On carbon paste or glassy carbon electrodes, AuNPs were assembled by casting colloid suspension, and were used for glucose oxidation [12] and $\mathrm{H}_{2} \mathrm{O}_{2}$ reduction [13]. ITO is another widely used conductive substrate for AuNP assembly. Multiple layers of AuNPs were obtained by infiltrating pre-synthesized nanoparticles into a polyelectrolyte film deposited on ITO. The electrode showed high electrocatalytic activity to the oxidation of nitric oxide [14]. Using the well established silane surface chemistry for ITO modification, dendrimer-capped Au nanoparticles were adsorbed on ITO for the electrocatalytic oxidation of cysteine and arsenite [15]. Oyama et al. devised a 2-step protocol for the in-situ chemical synthesis and deposition of AuNPs on ITO for the electrochemical investigation of nitric oxide, NADH, ascorbic acid, uric acid, and dopamine [16-18].

In the work described above, although the high electrocatalytic activity of AuNPs toward redox reactions of a number of chemical and biochemical molecules was demonstrated, the unique relationship of this activity with the nano-scale size of the material was not established. As with quantum dots, a nano-size related property is associated exclusively with the nanomaterials, i.e., it is not found in conventional bulk materials. The property displays sizedependence within the nanometer range. In all previously reported work, a few papers described direct comparison between AuNPs and bulk gold electrodes to demonstrate the unique electrocatalytic property of the nanoparticle $[6,8$, 10]. In all the other work, the observed catalysis may simply arise from the high activity of gold (not necessarily nano-sized gold) relative to the low activity base electrodes such as carbon and ITO. No dependence on nanoparticle size was investigated in any of the work. In this report, we investigated the electrocatalytic activity of AuNPs by attaching the pre-synthesized nanoparticles on ITO and examining the electrochemical response of dopamine. ITO is very inactive for dopamine oxidation, and thus minimizes interference to the response on gold $[17,18]$. Our results demonstrate that the observed electrocatalytic response of dopamine on AuNP is independent of the size and surface coverage of the nanoparticle, and is therefore ascribed to the material itself.

\section{Materials and methods}

\subsection{Reagents}

$\mathrm{NaBH}_{4}$, (3-aminopropyl)triethoxysilane (APTES), (3-Mercaptopropyl)triethoxysilane (MPTES), sodium citrate and hydrogen tetrachloroaurate (Ш) trihydrate $\left(\mathrm{HAuCl}_{4} \cdot 3 \mathrm{H}_{2} \mathrm{O}\right)$ were purchased from Sigma-Aldrich. All the other chemicals are of analytical reagent (A.R.) grade. Solutions were prepared in high-purity water from a Millipore milli-Q
(Biocel) water purification system (Billerica, MA, USA). Dopamine solution was prepared in phosphate buffer (20 mmol L $\left.{ }^{-1}, \mathrm{pH} 7.4\right)$.

\subsection{Apparatus}

Cyclic voltammetry was performed on a CHI 660B electrochemistry analyzer from $\mathrm{CH}$ Instruments (Austin, TX, USA) with a $\mathrm{Pt}$ counter electrode and an $\mathrm{Ag} / \mathrm{AgCl}\left(3 \mathrm{~mol} \mathrm{~L}^{-1} \mathrm{KCl}\right)$ reference electrode (also from $\mathrm{CHI}$ ). Scanning electron microscope (SEM) images were taken on a cold field emission SEM (JSM-6700F, JEOL Ltd. Tokyo, Japan). Sputtering of gold film on functionalized ITO surfaces was carried out on an EMITECH K550X sputter coater (Kent, UK).

\subsection{Synthesis of gold nanoparticles}

AuNPs with an average diameter of 6, 11, 21, 35 and $72 \mathrm{~nm}$ were prepared by reducing $\mathrm{HAuCl}_{4}$ with different amounts of citrate or a mixture of citrate and $\mathrm{NaBH}_{4}$ [19-22]. All glassware used for the preparation of AuNPs were thoroughly cleaned in freshly prepared aqua regia $\left(\mathrm{HNO}_{3}: \mathrm{HCl}\right.$ $=1: 3, V / V)$, rinsed extensively with deionized and high purity water, and then dried in an oven at $100{ }^{\circ} \mathrm{C}$ for $2-3 \mathrm{~h}$. A $20 \mathrm{~mL}$ solution of $0.01 \% \mathrm{HAuCl}_{4}$ was placed in a roundbottom flask fitted with a reflux condenser, and was brought to vigorous boiling while stirring. Different amounts of $1 \%$ citrate were then added to the refluxing $\mathrm{HAuCl}_{4}$ solution. For 11, 21, 35 and $72 \mathrm{~nm}$ AuNPs, 0.4, 0.3, 0.2 and 0.14 mL citrate were added, respectively. For $6 \mathrm{~nm}$ AuNPs, $0.2 \mathrm{~mL}$ citrate was added first and reacted for $1 \mathrm{~min}$, followed by addition of $0.2 \mathrm{~mL}$ of $0.375 \% \mathrm{NaBH}_{4}$ in $1 \%$ citrate. The solution was maintained at the boiling point with continuous stirring for about $15 \mathrm{~min}$. After the solution was allowed to cool to room temperature, the suspension was stored at $4{ }^{\circ} \mathrm{C}$ until further use. Assuming the spherical shape and a density equivalent to bulk gold $\left(19.30 \mathrm{~g} \mathrm{~cm}^{-3}\right)$, the concentration of AuNP suspension was calculated as follows: 44 $\mathrm{nmol} \mathrm{L}{ }^{-1}(6 \mathrm{~nm}), 7.2 \mathrm{nmol} \mathrm{L}{ }^{-1}(11 \mathrm{~nm}), 1.0 \mathrm{nmol} \mathrm{L}^{-1}(21$ $\mathrm{nm}), 0.22 \mathrm{nmol} \mathrm{L}^{-1}(35 \mathrm{~nm}), 0.026 \mathrm{nmol} \mathrm{L}^{-1}(72 \mathrm{~nm})$.

\subsection{ITO electrode modification}

ITO conductive glass (film thickness: $900 \pm 100 \AA$, conductivity: $19 \pm 2.1 \Omega \mathrm{m}^{-2}$ ) was obtained from Nanbo Optoelectronics Corporation (Shenzhen, Guangdong, China), and was cut into $2.5 \mathrm{~cm} \times 0.5 \mathrm{~cm}$ size slices. The slices were ultrasonically cleaned according to the published procedure, and then dried in an oven at $50{ }^{\circ} \mathrm{C}$ [23]. They were cleaned for $60 \mathrm{~s}$ with an Atmospheric Plasma Surface Treatment Systems CTD-1000z from Kulun Electronics Co., Ltd. (Nanjing, Jiangsu, China). The cleaned ITO electrodes were pretreated in $1 \% \mathrm{NH}_{4} \mathrm{OH}$ solution at $80{ }^{\circ} \mathrm{C}$ for $1 \mathrm{~h}$, leading to an active hydroxyl surface layer. The electrodes were immersed in a $2 \%(V / V)$ solution of APTES in dry toluene 
at room temperature for $4 \mathrm{~h}$ with shaking $(40 \mathrm{rpm})$. After the reaction, the slices were washed ultrasonically in dry toluene (5 min, twice) to remove physically adsorbed APTES from the surface, followed by anhydrous acetone and water (5 min, twice). The APTES modified ITO slices were dried under a stream of nitrogen $[24,25]$. They were stored in a sealed box till use. The modified ITO is denoted as ITO/APTES.

The deposition of $11 \mathrm{~nm}$ AuNPs was achieved by casting $20 \mu \mathrm{L}$ colloid solution to the surface of APTES modified ITO on an area of about $0.5 \mathrm{~cm} \times 0.5 \mathrm{~cm}$, and reacting for a certain period. Typically, the formation of a stable modification layer took at least $1,2,4,8$ and $24 \mathrm{~h}$ for the $6,11,21$, 35 and $71 \mathrm{~nm}$ AuNPs, respectively, which is attributable to the lower concentrations and small diffusion coefficients of the larger-sized AuNPs [25]. The resulting electrode was washed carefully with copious amount of water, and dried under a stream of nitrogen before use.

\subsection{Fabrication of regular gold electrodes}

Regular gold electrodes were prepared by sputtering gold film on silanized ITO substrates. ITO electrodes were cleaned as previously described. They were silanized in 5\% $(V / V)$ MPTES in dry toluene for $24 \mathrm{~h}$, washed and dried. The MPTES coating acts as a primer to improve the adhesion of Au film to the substrate [26]. About $40 \mathrm{~nm}$ thick gold film was deposited on the silanized ITO substrate by sputtering.

\section{Results and discussion}

To investigate the electrocatalytic property of AuNPs, ITO was selected as the conductive substrate for AuNP film deposition based on the fact that many organic compounds exhibit very sluggish electrochemical response on ITO [14-17]. In our work, AuNPs were synthesized by the wet chemistry method, and their size was varied by adjusting the amount of citrate in the reaction mixture [19-22]. AuNPs were attached to ITO substrates via well established silane surface chemistry with APTES [24, 25]. Figure 1 shows SEM image of $11 \mathrm{~nm}$ AuNPs deposited on ITO substrate. The nanoparticles are uniformly dispersed on the ITO surface and retained their original size and spherical shape. Adhesion of AuNPs to ITO is very strong and could withstand repeated rinse with water. Based on the particle number and diameter, the total surface area of gold is estimated to be about $0.21 \mathrm{~cm}^{2}$ on $0.25 \mathrm{~cm}^{2}$ ITO.

The deposited AuNPs were further characterized by cyclic voltammetry. As shown in Figure 2, no evident faradic current is observed on ITO/APTES electrodes in a phosphate buffer. However, after AuNP deposition, the electrode exhibits similar characteristic redox waves as a regular gold electrode, namely an oxidation peak at $\sim 1.05 \mathrm{~V}$ in the posi-

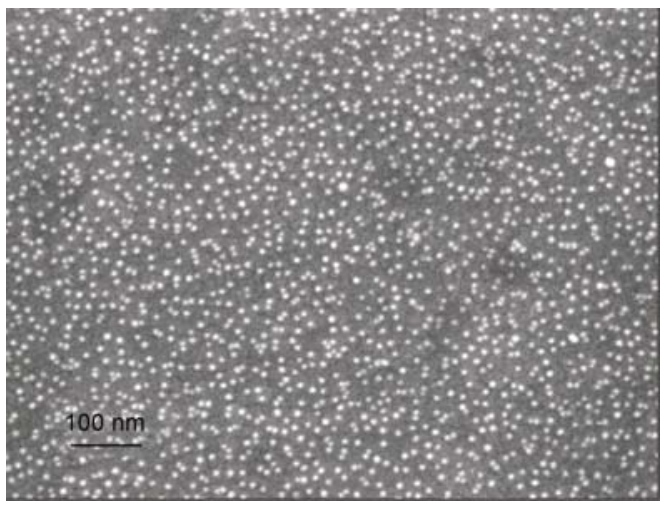

Figure 1 SEM image of $11 \mathrm{~nm}$ AuNPs deposited on ITO/APTES.

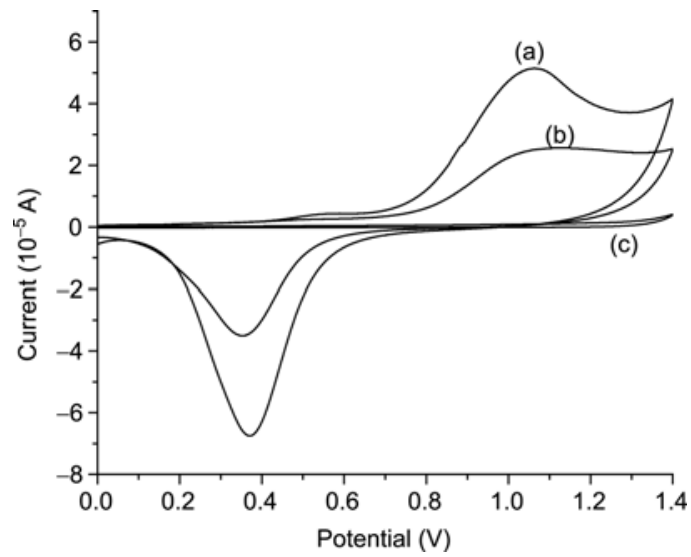

Figure 2 Cyclic voltammograms of (a) regular gold, (b) AuNPs (11 nm) on ITO/APTES, and (c) ITO/APTES electrode in PB solution $\left(20 \mathrm{mmol} \mathrm{L}^{-1}\right.$, $\mathrm{pH}$ 7.4). Scan rate: $50 \mathrm{mV} \mathrm{s}^{-1}$.

tive scan and a reduction peak at $\sim 0.35 \mathrm{~V}$ in the reverse scan. The voltammogram provides additional evidence that the AuNPs have been deposited on ITO, and indicates they are electronically conductive. From the reduction peak, the active surface area of the AuNPs is estimated as $0.16 \mathrm{~cm}^{2}$. This value is slightly smaller than the area estimated from the SEM image, suggesting that about $23 \%$ of the nanoparticles on ITO are electrochemically inactive. It is well known that silane surface chemistry usually produces rather inhomogeneous multi-layer films on ITO, resulting in a certain suppression of the charge transfer at the interface [27]. For those AuNPs deposited in the areas on ITO where the film is thick, it is plausible that the nanoparticles would be insulated by the nonconductive organic film.

Dopamine is selected as a model compound in our study because it is a very significant neurotransmitter, and its electrochemical response on ITO is negligible $[17,18]$. On a regular gold electrode, the oxidation of dopamine occurred at $0.3 \mathrm{~V}$ and the peak-to-peak separation was found to be $0.18 \mathrm{~V}$ at a scan rate of $50 \mathrm{mV} \mathrm{s}^{-1}$ (Figure 3(A)). The voltammogram is similar to that in previous reports [28]. Under identical conditions, dopamine was not oxidized on ITO/ APTES electrodes. However, once AuNPs were deposited 

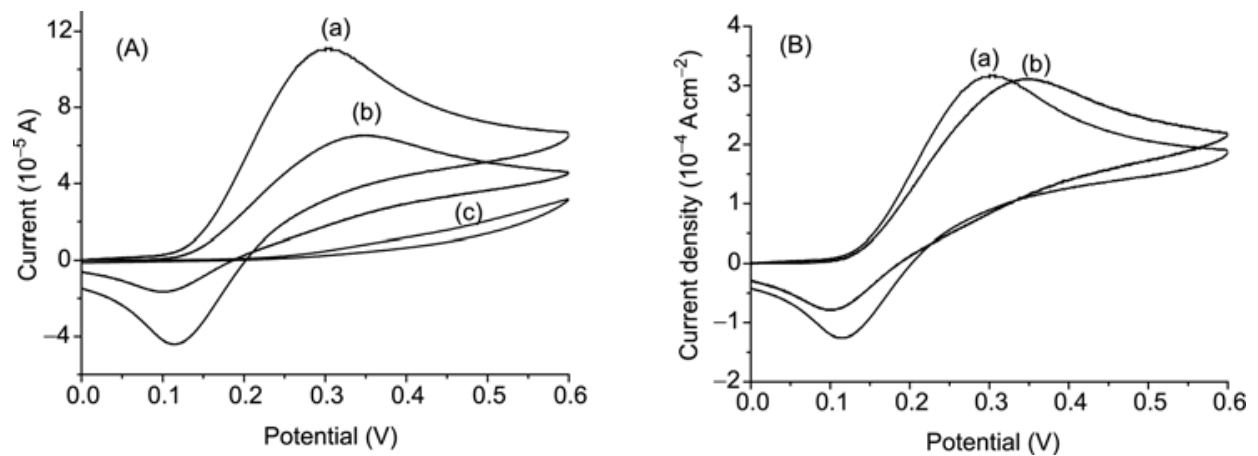

Figure 3 Cyclic voltammograms of $1 \mathrm{mmol} \mathrm{L}^{-1}$ dopamine solution ( $\mathrm{pH}$ 7.4) at a (a) regular gold; (b) AuNPs (11 nm) on ITO/APTES; and (c) ITO/APTES electrode. Scan rate: $50 \mathrm{mV} \mathrm{s}^{-1}$. The coordinate vertical scale is current in (A) and current density in (B), respectively.

on ITO, electrochemical response of dopamine emerged. Since the active area of regular gold electrode $\left(0.35 \mathrm{~cm}^{2}\right)$ is larger than that of AuNPs $\left(0.21 \mathrm{~cm}^{2}\right)$, current density was used to plot the voltammogram in Figure 3(B) for better comparison. The results show that the current density of dopamine oxidation is nearly the same on regular and AuNP electrodes. The oxidation peak moved positively to $0.35 \mathrm{~V}$ and the reduction peak moved negatively to $0.1 \mathrm{~V}$ on the AuNP electrode. Both current density and peak position suggest the AuNP electrode is not more active than the regular gold electrode. Additionally, the dependence of electrochemical current on potential scan rate was investigated. As shown in Figure 4, the anodic peak current of dopamine on the nanoparticle electrode increases linearly with the square root of scan rate $(r=0.994)$, which indicates a diffusion controlled oxidation process.

One of nano-material's unique characteristics is the size dependence of their chemical and physical properties. For example, semiconductor quantum dots exhibit fluorescence emission that is critically dependent on the size of the nanoparticle. To investigate the size dependence of electro-

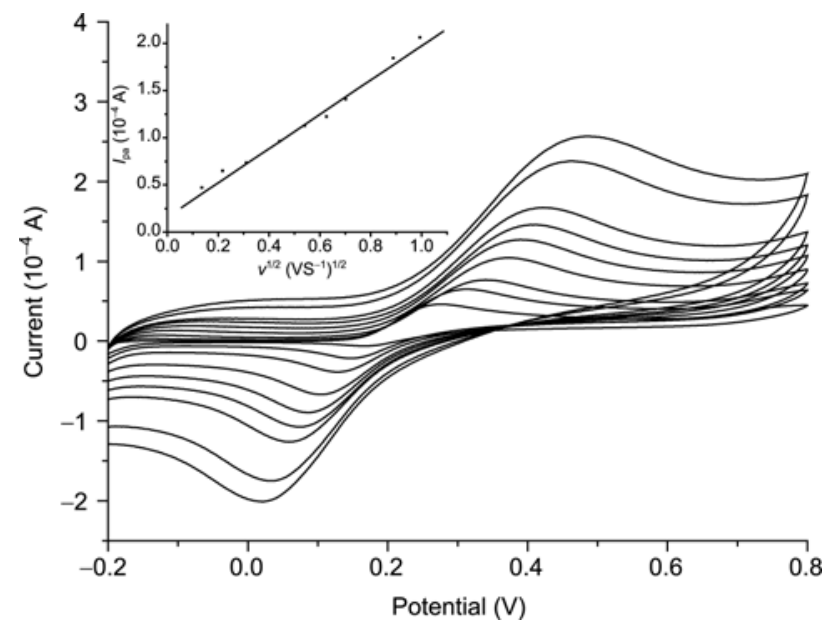

Figure 4 Cyclic voltammograms of $1 \mathrm{mmol} \mathrm{L}^{-1}$ dopamine solution at 11 nm AuNP electrode obtained at various scan rates. Scan rate: 20, 50, 100, 200, 300, 400, 500, 800 and $1000 \mathrm{mV} \mathrm{s}^{-1}$. Inset: The linear relationship between the anodic peak current and square root of the scan rate. catalytic activity of AuNPs, the electrochemical response of dopamine on AuNP electrodes of various sizes was examined. Figure 5 shows the SEM images of 6, 21, 35 and 72 $\mathrm{nm}$ AuNPs deposited on ITO/APTES electrodes. Similar to the $11 \mathrm{~nm}$ particles in Figure 1, these AuNPs were well dispersed on the electrode, and retained their shape and size. The image shows that the surface coverage of the larger particles is lower than the smaller ones. The total $\mathrm{Au}$ surface area on ITO was calculated to be $0.16,0.14,0.09$ and $0.065 \mathrm{~cm}^{2}$ for $6,21,35$ and $72 \mathrm{~nm}$ AuNP electrodes, respectively. In a dopamine solution, these electrodes displayed very different cyclic voltammograms (Figure 6). The voltammogram on $6 \mathrm{~nm}$ AuNP electrode is peak-shaped, whereas on 35 and $72 \mathrm{~nm}$ AuNP the curve is plateau at the top. Compared with the regular gold electrode in Figure 3, the oxidation peak shifted positively and maximum current decreased on all AuNP electrodes. After taking into account the difference in Au surface area, the current density was calculated to be $313,307,333$, and $354 \mu \mathrm{A} \mathrm{cm}^{-2}$ on $6,21,35$
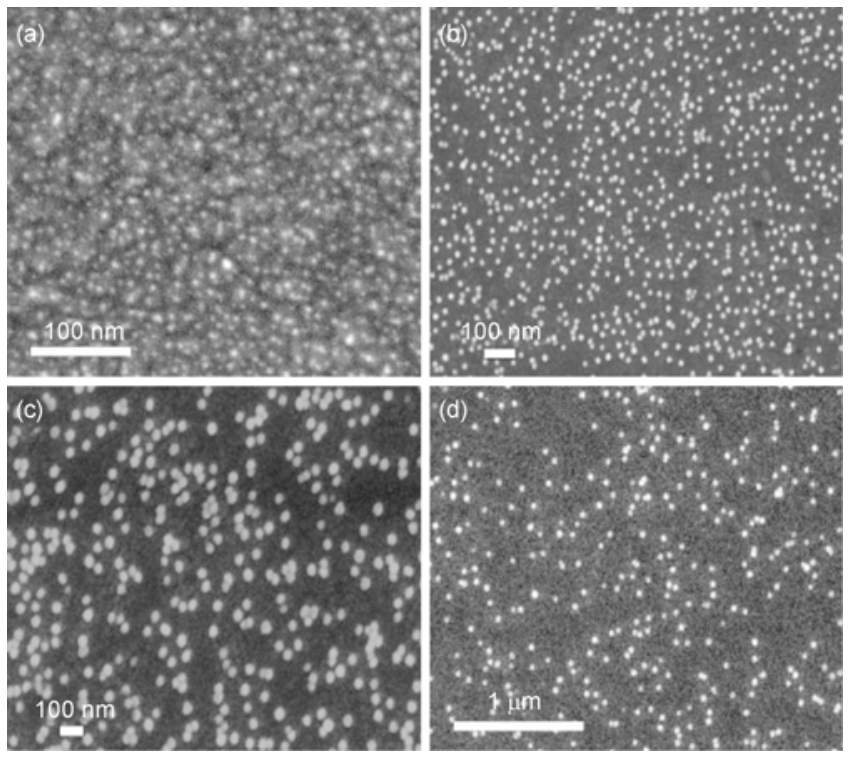

Figure 5 SEM of 6 (a), 21 (b), 35 (c) and (d) $72 \mathrm{~nm}$ AuNPs deposited on ITO/APTES electrode 


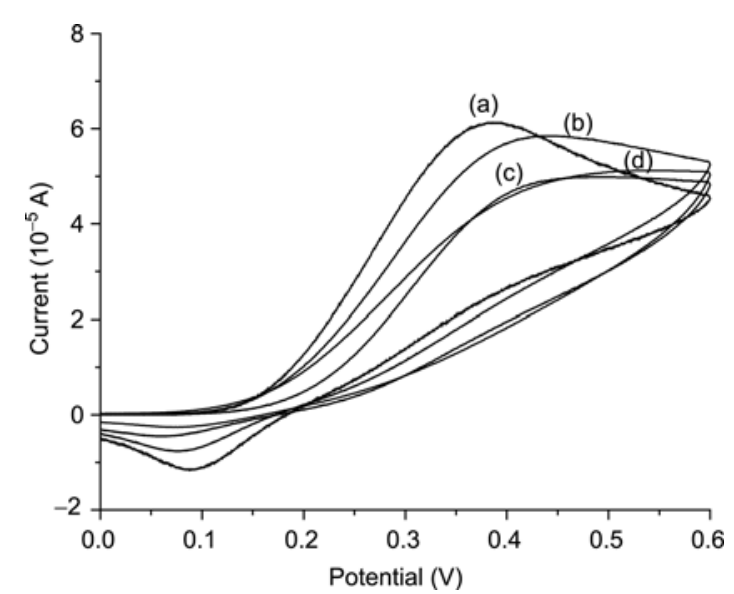

Figure 6 Cyclic voltammograms of $1 \mathrm{mmol} \mathrm{L}^{-1}$ dopamine solution on (a) 6, (b) 21 , (c) 35 , and (d) $72 \mathrm{~nm}$ AuNPs deposited on ITO/APTES electrode. Scan rate: $50 \mathrm{mV} \mathrm{s}^{-1}$.

and $72 \mathrm{~nm}$ AuNP electrodes, respectively. These values are slightly larger than the regular Au electrode $\left(290 \mu \mathrm{A} \mathrm{cm}^{-2}\right)$. Dopamine anodic peak potential and current density both suggest that the AuNP electrodes, regardless of the particle size, are not more active than the regular gold electrode.

The transition of voltammograms in Figure 6 from peak to sigmoidal shape as the particle size is increased coincides with the reduced surface coverage of the particle on ITO. It was suspected that the transition reflected a change in the mode of mass diffusion of dopamine to the electrode. On AuNP electrodes with relatively low surface coverage, each nanoparticle acts as an independent nano-electrode. Mass diffusion is predominantly radial, producing sigmoidal voltammogram. At high surface coverage, however, the distance between neighboring particles is shortened. The diffusion zone around each particle overlaps with others and becomes linear, resulting in peak-shaped voltammogram.

To support this rationale, ITO electrodes deposited with AuNPs of the same size but different surface coverage were prepared and investigated. First, a stock solution of $11 \mathrm{~nm}$ AuNPs was diluted to $1 / 2,1 / 4,1 / 8,1 / 20$ with high-purity water. They were cast separately on ITO/APTES to fabricate the nanoparticle electrodes. SEM images in Figure 7 show the surface coverage of $11 \mathrm{~nm}$ AuNPs on ITO decreased as the colloid was diluted. According to the calculation, total surface areas of gold on ITO are 0.16, 0.13, 0.077 and $0.021 \mathrm{~cm}^{2}$. These electrodes were employed in the cyclic voltammetric measurement of dopamine. Voltammograms in Figure 8 demonstrate apparent dependence on the electrodes used. As the particle coverage on ITO surface was decreased, the curve changed from peak-shaped to sigmoidal, and the oxidation maximum is shifted toward more positive potentials (from $0.32 \mathrm{~V}$ to $0.74 \mathrm{~V}$ ). This change is very similar to what was observed in Figure 6 for AuNP electrodes of different sizes, and could be rationalized by the transition in mass diffusion mode.
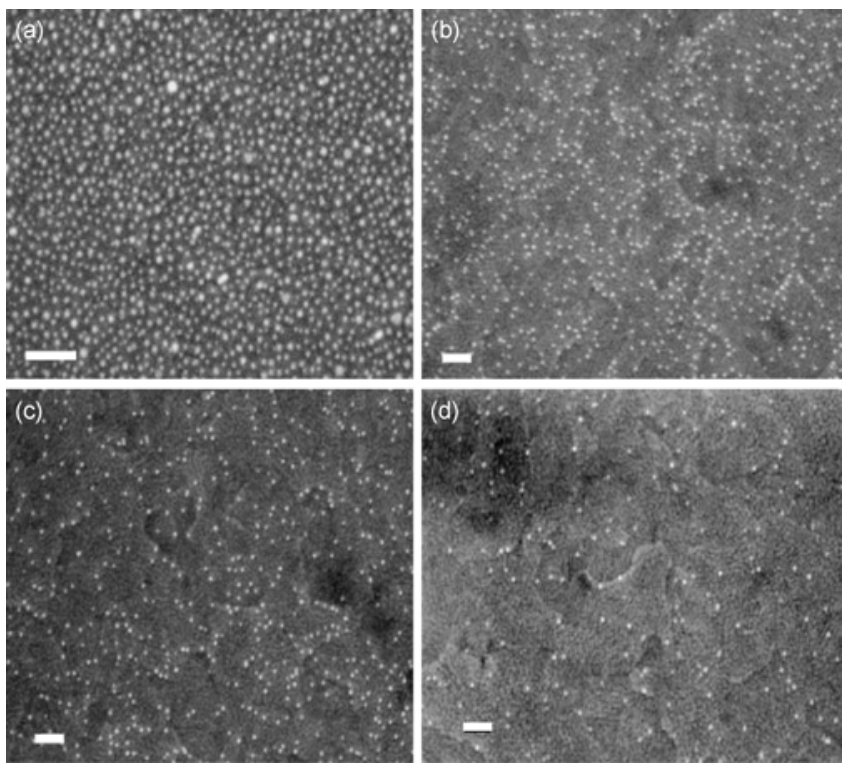

Figure 7 SEM images of ITO/APTES electrodes deposited with different concentrations of $11 \mathrm{~nm}$ AuNP colloid. (a) 3.6, (b) 1.8, (c) 0.9, and (d) 0.36 nmol L ${ }^{-1}$. Scale bar: $100 \mathrm{~nm}$.

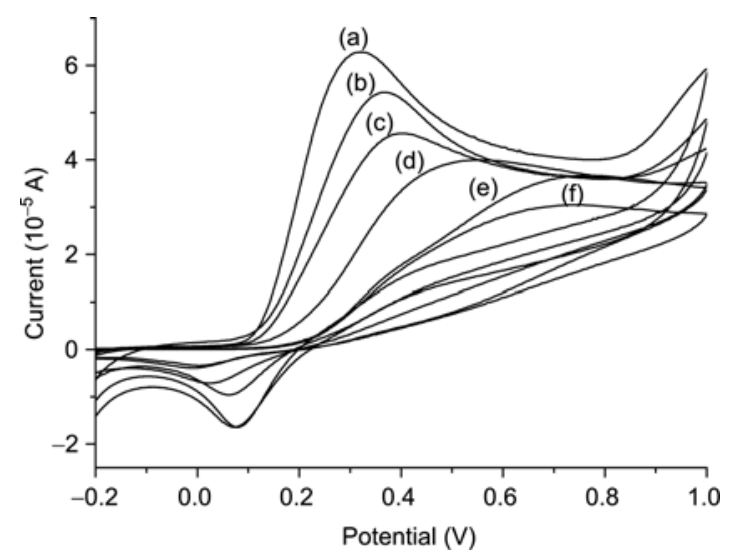

Figure 8 Cyclic voltammograms of $1 \mathrm{mmol} \mathrm{L}^{-1}$ dopamine solution on ITO/APTES electrodes deposited with different concentrations of $11 \mathrm{~nm}$ AuNP colloid. (a) 7.2, (b) 3.6, (c) 1.8 , (d) 0.9 , (e) $0.36 \mathrm{nmol} \mathrm{L}^{-1}$, and (f) no AuNP. Scan rate: $50 \mathrm{mV} \mathrm{s}^{-1}$.

\section{Conclusions}

This work investigated the electrochemical reaction of dopamine on AuNP modified ITO electrodes. AuNPs were attached to silanized ITO surface, which suppressed dopamine electrochemical oxidation on ITO itself and permitted examination of AuNPs with minimal interference. Significantly enhanced electrochemical response of dopamine was observed on AuNP electrodes. However, the response is at best as reversible as the regular bulk Au electrode, regardless of the size of AuNP and its surface coverage on ITO. It is therefore concluded that there is no nanometer size effect of AuNP modified ITO on the electrochemistry of dopamine. 
The authors express their great thanks for the support from the National Natural Science Foundation of China (90813016, 20825519 \& 20921063).

1 Luo XL, Morrin A, Killard AJ, Smyth MR. Application of nanoparticles in electrochemcial sensors and biosensors. Electroanalysis, 2006, 18: 319-326

2 Welch CM, Compton RG. The use of nanoparticles in electroanalysis: a review. Anal Bioanal Chem, 2006, 384: 601-619

3 Guo S, Wang E. Synthesis and electrochemical applications of gold nanoparticles. Anal Chim Acta, 2007, 598: 181-192

4 You T, Niwa O, Tomita M, Hirono H. Characterization of platinum nanoparticle-embedded carbon film electrode and its detection of hydrogen peroxide. Anal Chem, 2003, 75: 2080-2085

5 You T, Niwa O, Chen Z, Hayashi K, Tomita M, Hirono S. An amperometric detector formed highly dispersed Ni nanoparticles embedded in a graphite-like carbon film electrode for sugar determination. Anal Chem, 2003, 75: 5191-5196

6 El-Deab MS, Ohsaka T. An extraordinary electrocatalytic reduction of oxygen on gold nanoparticles electrodeposited gold electrodes. Electrochem Commun, 2002, 4: 288-292

7 Raj CR, Okajima T, Ohsaka T. Gold nanoparticle arrays for the voltammetric sensing of dopamine. J Electroanal Chem, 2003, 543: 127-133

8 Wang L, Bai J, Huang P, Wang H, Zhang L, Zhao Y. Self-assembly of gold nanoparticles for the voltammetric sensing of epinephrine. Electrochem Commun, 2006, 8: 1035-1040

9 Su L, Mao L. Gold nanoparticle/alkanedithiol conductive films selfassemble onto gold electrode: Electrochemistry and electroanalytical application for voltammetric determination of trace amount of catechol. Talanta, 2006, 70: 68-74

10 Raj CR, Abdelrahman AI, Ohsaka T. Gold nanoparticle-assisted electroreduction of oxygen. Electrochem Commun, 2005, 7: 888-893

11 Jena BK, Raj CR. Ultrasensitive nanostructured platform for the electrochemical sensing of hydrazine. J Phys Chem C, 2007, 111: 6228-6232

12 Tominaga M, Shimazoe T, Nagashima M, Taniguchi I. Electrocatalytic oxidation of glucose at gold nanoparticle-modified carbon electrodes in alkaline and neutral solutions. Electrochem Commun, 2005, 7: 189-193

13 Chandra D, Jena BK, Raj CR, Bhaumik A. Functionalized mesoporous cross-linked polymer as efficient host for loading gold nanoparticles and its electrocatalytic behavior for reduction of $\mathrm{H}_{2} \mathrm{O}_{2}$. Chem Mater, 2007, 19: 6290-6296

14 Yu A, Liang Z, Cho J, Caruso F. Nanostructured electrochemical sensor based on dense gold nanoparticle film. Nano Lett, 2003, 3: 1203-1207
15 Ca DV, Sun L, Cox JA. Optimization of the dispersion of gold and platinum nanoparticles on indium tin oxide for the electrocatalytic oxidation of cysteine and arsenite. Electrochim Acta, 2006, 51: 2188-2194

16 Zhang J, Oyama M. Gold nanoparticle arrays directly grown on nanostructured indium tin oxide electrodes: characterization and electroanalytical application. Anal Chim Acta, 2005, 540: 299-306

17 Zhang J, Oyama M. Electrocatalytic activity of three-dimensional monolayer of 3-mercaptopropionic acid assembled on gold nanoparticle arrays. Electrochem Commun, 2007, 9: 459-464

18 Goyal RN, Gupta VK, Oyama M, Bachheti N. Gold nanoparticles modified indium tin oxide electrode for the simultaneous determination of dopamine and serotonin: application in pharmaceutical formulations and biological fluids. Talanta, 2007, 72: 976-983

19 Frens G. Controlled nucleation for the regulation of the particle size in monodisperse gold suspensions. Nat Phys Sci, 1973, 241: 20-22

20 Zhong Z, Patskovskyy S, Bouvrette P, Luong JHT, Gedanken A. The surface chemistry of au colloid and their interactions with functional amino acids. $J$ Phys Chem B, 2004, 108: 4046-5052

21 Nath N, Chilkoti A. A colorimetric gold nanoparticle sensor to interrogate biomolecular interactions in real time on a surface. Anal Chem, 2002, 74: 504-509

22 Grabar KC, Allison KJ, Baker BE, Bright RM, Brown KR, Griffith FR, Fox AP, Keating CD, Musick MD, Natan MJ. Two-dimensional arrays of colloidal gold particles: a flexible approach to macroscopic metal surfaces. Langmuir, 1996, 12: 2353-2361

23 Li C, Liu SL, Guo LH, Chen DP. A new chemically amplified electrochemical system for DNA detection in solution. Electrochem Commun, 2005, 7: 23-28

24 Hedges DHP, Richardson DJ, Russell DA. Electrochemical control of protein monolayers at indium tin oxide surfaces for the reagentless optical biosensing of nitric oxide. Langmuir, 2004, 20: 1901-1908

25 Chen Z, Zu Y. Gold nanoparticle-modified ito electrode for electrogenerated chemiluminescence: well-preserved transparency and highly enhanced activity. Langmuir, 2007, 23: 11387-11390

26 Goss CA, Charych DH, Majda M. Application of (3-mercaptopropyl) trimethoxysilane as a molecular adhesive in the fabrication of vapor-deposited gold electrodes on glass substrates. Anal Chem, 1991, 86: $85-88$

27 Hillebrandt H, Tanaka M. Electrochemical characterization of selfassembled alkylsiloxane monolayers on indium-tin oxide (ito) semiconductor electrodes. J Phys Chem B, 2001, 105: 4270-4276

28 Zhang J, Kambayashi M, Oyama M. Seed mediated growth of gold nanoparticles on indium tin oxide electrode: electrochemical characterization and evaluation. Electroanalysis, 2005, 17: 408-416 